zwischen den Parteien besteht. Wenn irgend eine vernünftige Wahrscheinlichkeit für ein Utbereinkommen besteht, so sollen Schritte zur Anbahnung unternommen und inzwischen die Úberweisung an das Gericht vertagt werden (Vorschrift 73). Erst wenn keine Aussicht auf eine Einigung besteht, wird der Antrag an das Gericht weitergegeben.

b) Nach 4 Jahren nach Anmeldung des Patentes (jedoch nicht vor dem 28. August 1908) kann man beim Comptroller die Zurücknahme beantragen, weil der patentierte Artikel oder das patentierte Verfahren ausschlieBlich oder hauptsächlich außerhalb Englands hergestellt oder ausgeführt wird (Abschnitt 27 des Gesetzes). Der Patentinhaber soll innerhalb 14 Tage von der Absendung des Duplikatantrages oder innerhalb einer weiteren vom Comptroller zu gewährenden Frist durch beglaubigte Erklärungen feststellen, ob die im Antrage gemachten Angaben zutreffen. Falls sie unzutreffend sind, soll angegeben werden, in welehem Umfange sie unzutreffend sind. Es soll auch angegeben werden, wo der patentierte Gegenstand in Großbritannien hergestellt oder das Verfahren ausgeführt wird. Wenn keine Ausführung der Erfindung in Großbritannien erfolgt ist, sollen die Gründe für die Unterlassung dargelegt werden.

Das weitere Verfahren ist dem Einspruchsverfahren analog.

Die beiden Zurücknahmegründe sind nicht nur in ihrem Wesen und der Verfahrensvorschrift verschieden, sondern zeigen auch eine verschiedene Stellung der entscheidenden Behörde gegen den Patentinhaber. Bei dem Antrage auf Zwangslizenz streben die Vorschriften augenscheinlich eine Vermeidung der Zurücknahme und überhaupt der richterlichen Entscheidung an. Die Vorschrift, daß das Handelsamt sich um die Herbeiführung einer Einigung bemühen soll, deutet wohl klar auf die Tendenz der Bestimmung hin. Gänzlich anders mutet dagegen das Verfahren wegen Nichtausübung an. Aus diesen Bestimmungen spricht jedenfalls keine Milde. Man wird also damit zu rechnen haben, daß ein Ausführungszwang in Großbritannien besteht.

\section{Chemische Lichtwirkungen.}

\section{Von Hans Stobbe, Leipzig.}

Auszug aus einem Vortrage, rehaiten in der Hauptverbammlung des Bezirksvereins Sachsen-Anhalt in Magdeburg am 17. November 1907.

(Eingeg. den 9./1. 1908.)

Zu den chemischen Lichtwirkungen gehört vor allen Dingen der AssimilationsprozeB, jene hochwichtige physiologisch-chemische Reaktion, bei der im Pflanzenkörper hochmolekulare organische Stoffe aus Kohlensäure und Wasser aufgebaut werden. Ferner gehören bierher auch eine sehr große Zahl anderer Zustandsänderungen, rein chemische Vorgänge, die ebenfalls entweder nur durch das Licht hervorgerufen oder doch wenigstens in ihrem zeitlichen Verlaufe beeinflußt werden.

Die ersten Beobachtungen über die Beteiligung des Lichtes bei solchen Prozessen reichen bis in das Altertum und das Mittelalter zurück. Das Grünen der Pflanzen, die Bräunung der menschlichen Haut, das Bleichen von Leinen, die Entstehung des Purpurfarbstoffes aus dem schleimigen, farblosen oder gelblichen Safte einiger Schnecken des Mittelmeeres, die Veränderungen anorganischer Farbstoffe, z. B. des Zinnobers, werden bereits in diesen Zeitepochen auf die Wirkung des Lichtes zurückgeführt.

Nachdem dann die Alchymisten in den späteren Zeitaltern nur höchst spärliche Beiträge zur Photochemie geliefert haben, begann erst vom 18. Jahrhundert an ein systematisches Studium der chemischen Lichtwirkungen. Man untersuchte in erster Linie anorganische Stoffe, vornehmlich die Salze der Schwermetalle, auf ihr Verhalten gegen Licht; man erkannte, daß die Entfärbung einer alko. holischen Eisenchloridlösung bei Luftabschluß durch das Licht bewirkt wurde, und daß die ursprüngliche Farbe dieser Flüssigkeit im Dunklen und bei Luftzutritt wiederkehrte. Man erforschte den Grund für den Farbwechsel der mit Berliner Blau gefärbten Stoffe bei Licht und Dunkelkeit. Die Lichtempfindlichkeit der Silbersalze entdeckte ein deutsoher Arzt, Joh an $\mathbf{n}$ Hein rich Schulze, im Jahre 1727; er war der erste, welcher Schriftzüge in Gestalt von Sehablonen auf mit Höllensteinlösung getränkten Papieren wiederzugeben verstand. Nach $\mathrm{S}$ ch u l ze beschäftigten sich viele andere Forscher mit den Silbersalzen, unter ihnen vornehmlich Sch e e le. Fr stellte sicn bei seinen photochemischen Studien zum ersten Male die sehr wichtige Frage: Wenn die Stoffe durch das Licht verändert werden, welcher Art sind die Veränderungen, und woraus besteht die Materie nach der Belichtung? S c h e e le fand z. B., daß das bei der Insolation des Chlorsilbers entstehende schwarze Pulver metallisches Silber enthielt und daB außer diesem auch Salzsäure entstände, wenn die Belichtung unter Wasser vorgenommen würde. Ferner konstatierte derselbe Forscher, daB ein mit Chlorsilber bestrichener Papierstreifen bei der Einwirkung des zerlegten Sonnenlichtes nicht gleichmäßig dunkelt, sondern daß dieses Salz im roten, gelben und grünen Teile des Spektrums fast unverändert bleibt, im blauen und violetten Lichte hingegen schnell zersezt wird.

Dieses staunenerregende Ergebnis sollte bald noch übertroffen werden durch $R$ it te $r \&$ Entdeckung der ultravioletten Strahlen (1801) und durch die Tatsache, daß die Silbersalze gerade in diesen dem Auge unsichtbaren Strahlen ebenfalls und besonders stark verändert werden. Und als dann weiter gezeigt wurde, daß die vielen anderen lichtempfindlichen Stoffe sich den Spektralfarben gegenüber ähnlich verhielten, verallgemeinerte man die bisher gewonnenen Resultate und teilte die Farben resp. die ihnen entsprechenden Strahlen in zwei Kategorien - in diejenigen, welche chemische Wirkungen hervorriefen, und solche, welche diese Fähigkeit nicht zeigten. Die , c h e $\mathrm{m}$ is o h wirks am e $\mathrm{n}^{\text {" }}$ seien allein die brechbarsten, die blauen, violetten und ultravioletten Strahlen.

Wie voreilig und wie wenig zutreffend diese zum Teil noch bis auf den heutigen Tag beibehaltene Einteilung war, zeigten weitere photochemische Versuche an anderen Stoffen, vornehmlich an farbigen Substanzen, z. B. Bleioxyd, Eisenvitriol. 
Diese blieben nicht nur nicht unter den ,sogenannten chemisch wirksamen" Strahlen intakt, sondern wurden gerade unter rotem Lichte schneller verändert, als unter anderen Teilen des Spektrums. Alle diese Tatsachen fanden später ihre befriedigende Erklärung in einem von $G r o t t h u s$ a $u f$ gestellten Gesetze, nach welchem ein Stoff nur durch diejenigen Strahlen chemisob verändert wird, die von ihm selbst absorbiert werden. Dieses Gesetz, das heute die ganze Photochemie beherrscht, besagt also, daß einer chemischen Lichtwirkung erst eine Absorption der Lichtstrahlen, eine Aufnahme von strahlender Energie voraufgehen muB. Und da ein weißer Stoff ein solcher ist, der ultraviolette Strahlen verschluckt, und da ferner im allgemeinen ein roter Stoff im grünen, ein gelber im violetten, ein grüner im roten, ein blauer im orangefarbenen Spektralgebiete absorbiert, so folgt hieraus, daß, wenn farblose oder farbige Stoffe überhaupt lichtempfindlich sind, sie immer nur in einem speziellen aber verschiedenen Bezirke des Spektrums reagieren können. Die Bezeichnung, ,chemisch wirksame Strahlen" ist daher nicht auf eine einzige Lichtgattung, auf das Ultraviolett allein, anzuwenden, sondern gilt allgemein für alle Lichtgattungen. Jeder lichtempfindliche Stoff hat je nach seiner Farbe und Absorptiensvermögen seine eigene Gruppe chemisch wirksamer Strahlen. -

Ebenfalls bis in das 18. Jahrhundert zurück datiert das Studium des Assimilations prozesses der Pflanzen. Nachdem Priest. le y 1772 nachgewiesen hatte, $\mathrm{da} \beta$ die grünen Pflanzenteile den für die Atmung der Menschen und Tiere notwendigen Sauerstoff aus der Kohlensäure der Luft produzieren, und nachdem Ing en hou s z 1783 zeigte, daß für diese Reaktion die Mitwirkung des Lich tes unbedingt erforderlich sei, stellte S e n e bier auf Grund seiner eigenen und $d$ e $S$ a $\mathrm{s}$ s ures Arbeiten eine Theorie des Assimilationsprozesses auf, in der nicht nur die Sauerstoffproduktion, sondern auch das eigene Wachstum der Pflanzen mit der Zerlegung der Kohlensäure in Verbindung gebracht wird. S e n e b i e r s Theorie lautet in moderner Ausdrucksweise foldengermaßen : Die lebende Pflanze baut ihre organische Substanz auf aus Kohlensäure und Wasser in den grünen Pflanzenteilen unter dem Einflusse des Lichtes. Dieser Satz ist die Basis geworden, auf die sich das weitere Studium dieses hochwichtigen Prozesses im folgenden 19. Jahrhundert stützt.

Wollen wir seine Gültigkeit prüfen, so stoßen wir bei der Mannigfaltigkeit der die Reaktion bedingenden Faktoren auf eine große Fülle von Fragen, die experimentell gelöst werden mußten, und von denen einige hier kurz gestreift werden sollen. Die erste Frage wäre: $W$ el ches ist das erste Produkt der pflanzlichen As s im ilation? Tatsache ist, daß in der Regel als niedrigst molekulare Assimilierungsprodukte die Zucker der Hexosegruppe auftreten, die dann weiter in Stärke verwandelt werden. Diese beiden recht kompliziert zusammengesetzten Kohlehydrate können nun aber nicht wohl direkt aus der Kohlensäure entstehen. Und so hat denn schon vor mehr als drei Jahrzehnten A. v. B a e y e r die Hypothese aufgestellt, daß das Wasser zunächst die Kohlensäure zu Formaldehyd reduziere, daß es selbst zu Sauerstoff oxydiert werde

$$
\mathrm{CO}_{2}+\mathrm{H}_{2} \mathrm{O}=\mathrm{CH}_{2} \mathrm{O}+\mathrm{O}_{2} \text {, }
$$

und daß der Formaldehyd der eigentliche Baustein für die Photosynthese der höher molekularen Verbindungen sei.

Diese Hypothese gewann sehr an Wahrscheinlichkeit, als es gelang, die Zucker und zuckerähnliche Substanzen aus Formaldehyd und anderen niedermolekularen Aldehyden synthetisch darzustellen; sie harrt aber noch des direkten Beweises, insofern, als bisher alle Versuche, den Formaldehyd im Pflanzenorganismus aufzufinden, vergeblich gewesen sind.

Früher glaubte man allgemein, daß auch für den Aufbau der EiweiBstoffe das Licht erforderlich sei. Dem scheint aber nicht so zu sein, da eine große Reihe von Forschern, namentlich $P$ f e f f e $r$, nachgewiesen haben, daB die Blätter Proteinstoffe auch im Dunkeln bilden können, und $\mathrm{da} ß \mathrm{zu}$ ihrer Synthese außer Nitraten nur eine erhebliche Menge löslicher Kohlehydrate vorhanden sein müsse. Und das, was für die Bildung der Stärke und der Eiweißkörper gilt, wird wohl auch für die vielen anderen im Pflanzenkörper produzierten organisohen Stoffe gelten, nämlich, daß sie teils mit, teils ohne Mitwirkung des Lichtes entstehen.

Eine zweite Frage wäre: Ist den $\mathbf{n}$ d i e Photosynthese des Zuckers und der Stärke andie lebende Zelle gebun den ? Auch hierüber liegen Untersuchungen, allerdings erst aus jüngster Zeit, vor. Man hat aus grünen Blättern Glycerinextrakte hergestellt, die kein Protoplasma, wohl aber den grünen Pflanzenfarbstoff und Enzyme, leblose, bisher wenig charakterisierte Stoffe enthielten. Zeitweise glaubten einige Forscher, mit diesem Extrakte aus kohlensäurehaltigem Wasser unter Mitwirkung des Lichtes Sauerstoff und sogar Formaldehyd gewonnen zu haben. Jedoch scheinen diese Versuche und deren Ergebnisse nicht ganz einwandfrei zu sein, da sie teils von denjenigen Forschern, die über sie berichtet haben, widerrufen wurden, teils noch nicht genügend nachgeprüft worden sind. Es bleibt daher augenblicklich nur übrig, die weitere Entwicklung dieser Angelegenheit abzuwarten. Sollte sich jedoch das Problem der Photosynthese der Kohlehydrate ohne Protoplasma in Zukunft realisieren lassen, so würden wir eine Entdeckung von fundamentaler Bedeutung vor uns haben, etwa ein Analogon zu dem $B$ u c h $n$ e r schen Verfahren, den Zucker ohnè Mitwirkung der lebenden Hefenzelle, lediglich durch deren Enzym, die leblose Zymase, zu Alkohol und Kohlensäure zu vergären.

Eine dritte Frage wäre : W e n n n a c h g e wiesen ist, daB das Licht fürden AssimilierungsprozeBerforderlich ist-istesin seinerGesamtmenge odernurzum Teil dabei beteiligt? Die Untersuchungen hierüber sind sehr zahlreich. Sie stimmen alle darin überein, daß das Maximum der Assimilation im gelben und roten Lichte vor sich geht, während unter blauem Lichte das Wachs- 
tum, besonders die Entwicklung der Fortpflanzungsorgane in außerordentlichem Grade beeinträchtigt werden. Hieraus folgt also, daß nur ein sehr kleiner Teil der Spektralfarben bei der Photosynthese beteiligt ist. Und diese Erkenntnis führt zu der weiteren sehr wichtigen Frage : W e s h a l b erfolgt die Assimilierung nurinden grünen Pflanzenteilen? Welchen Anteil hat der grüne Farbstoff, das Chlorophyll, bei der Reaktion? Wir erinnern nur an das Grot th u s sche Gesetz, nach welchem nur diejenigen Strahlen chemisch wirksam sind, die absorbiert werden. Derjenige Stoff, der rote und gelbe Strahlen absorbiert, ist ebon das Chlorophyll; dieses oder ein ihm ähnlich absorbieronder Stoff ist also unbedingt erforderlich für die Lichtsynthese. Die Wirkungsweise des Chlorophylls ist noch nicht endgültig aufgeklärt. Man kann aber hierfür zwei Hypothesen aufstellen. Nach der ersten vereinigt sich das Chlorophyll nach der Asborption der Strahlen mit Kohlensäure und Wasser zu lockeren chemischen Verbindungen, die dann im weiteren Verlaufe des Prozesses unter Rückbildung des freien Chlorophylls zu Formaldehyd und Sauerstoff zerfallen. Nach der zweiten Hypothese negiert man die chemische Vereinigung der Reaktionsprodukte; man beschränkt sich darauf, anzunehmen, daß das Chlorophyll das rote und gelbe Licht verschlucke, $\mathrm{da} B$ es dieses in Lieht von anderer Wellenlänge, z. B. ultraviolettes Licht, umforme, welches dann seinerseits von dem farblosen Gemenge von Kohlensäure und Wasser absorbiert werde und diese dann zur Reaktion bringe. Ohne sich für die eine oder die andere Hypothese zu entscheiden, kann man die Mitwirkung des Chlorophylls bei der Photo. synthese als eine katalytische bezeichnen; das Chlorophyll ist ein Lichtkatalysator des Assimilationsprozesses. Diese Auffassung gewinnt sehr an Wahrscheinlichkeit, wenn man die Betätigung des Chlorophylls vergleicht mit der Wirkung der o $p$ tischen Sensibilisatorender orthoehromatischen Photographie. Auch diese von H. W. Vogel in die photographische Praxis eingeführten Substanzen - meist blaue, violette, purpurne Farbstoffe - sind Katalysatoren für den Zersetzungsprozeß des Bromsilbers durch langwellige Strahlen. Thre Wirkungsweise kann nun wieder in doppelter Weise gedeutet werden. Nach der ersten Deutung absorbieren die sensibilisierenden Farbstoffe zunächst die langwelligen (roten und gelben) Strahlen, um sie in die kurzwelligen (die blauen, violetten und die ultravioletten) Strahlen zu transformieren; die letzteren werden dann ihrerseits von dem farblosen Silberbromid absorbiert und veranlassen dessen Zersetzung. Nach der zweiten Deutung vereinigen sich die Farbstoffe mit dem Bromsilber zunächst zu chemischen Verbindungen, die dann nach der $\mathrm{Ab}$ sorption der gelben und roten Strahlen in Silbersubbromid, Brom, die Farbstoffe selbst oder deren Umwandlungsprodukte zerfallen. Diese letztere Auffassung soheint gestützt zu werden durch die Beobachtung von Eder und Valenta, nach welcher nicht etwa alle dunklen Farbstoffe sensibilisieren, sondern nur solche, die sich innig mit dem Bromsilberkorn vereinigen und dieses wirklich färben.
Wir haben aus diesem Vergleiche den Parallelismus zwischen den Sensibilisatoren der photographischen Platte und dem Lichtkatalysator der Photosynthese erkannt und haben auch aus den übrigen voranstehenden Darlegungen gesehen, welche Faktoren für den Assimilierungsprozeß maßgebend sind, und wie diese allergrößte aller photochemischen Reaktionen ohne das Licht, ohne die Sonne, nicht vonstatten gehen kann.

Die Sonne ist die Spenderin der Energie für diesen Prozeß. Man hat den Energiebetrag berechnet, der von der Sonne täglich der von grünen Pflanzen bestandenen Erdoberfläche geliefert wird, und hat gefunden, daß dieser Betrag bei aller seiner Größe doch nur einen verschwindend kleinen Bruchteil ausmacht von der gesamten Lichtenergie, die dauernd der ganzen Erdoberfläche zugeführt wird. Der allergrößte Teil der Sonnenenergie geht also ungenutzt verloren.

Angesichts dieser letzteren Tatsache scheint es nicht nur zweckmäßig, sondern sogar notwendig, sich heutzutage noch viel mehr wie bisher mit photochemischen Studien der allerverschiedensten Art zu befassen. Man soll versuchen, die uns umsonst in solcher Fülle dargebotene strahlende Energie in irgend welcher Weise nutzbar zu machen. Was ist bisher in dieser Richtung geschehen?

Das ganze 19. Jahrhundert hat außer dem bereits Erwähnten manches Neve auf photochemischem Gebiete gebracht, aber doch nur verhältnis. mäßig wenig im Vergleiche zu den zahlreichen Errungenschaften, die die Forschungen auf anderen rein chemischen und physiko-chemischen Gebieten zutage gefördert haben. Nur wenige Chemiker - an ihrer Spitze Buns en - beschäftigten sich um die Mitte des vergangenen Jahrhunderts mit photochemischen Studien, und unter ihnen bei weitem die Mehrzahl mit der Photographi e, dieser bisher fast einzigen praktischen Verwertung photochemischen Forschens. Man hat die Technik der Bilderzeugung in grauen, braunen und schwarzen Farbentönen zu einer großen Vervollkommnung ausgebildet und hat es neverdings sogar erreicht, auch die Bilder der uns umgebenden Welt in ihren natürlichen Farben direkt auf der Platte der photographischen Camera ontstehen zu lassen nach Verfahren, die im Prinzipe teilweise schon zu Anfang des 19. Jahrhunderts erkannt worden waren.

Aber nicht allein diese auf der Zersetzung der Silbersalze beruhenden Lichtwirkungen, sondern auch andere photochemische Prozesse werden in den verflossenen letzten drei Dezennien studiert, insbesondere von seiten der Farbenchemiker. Sie untersuchen die $f a r b i g$ en $S$ t of $f$ e, die zum Färben unserer Gebrauchsgegenstände und ihrer Rohmaterialien Verwendung finden, auf ihr Verhalten gegen Licht.

Alle Farbst of $\mathrm{fe}$ werden durch das lieht beeinflußt, aber glïcklicherweise in ganz außerordentlich verschiedenem Grade. Wir kennen einerseits sehr lichtechte, die selbst in Monaten und Jahren kaum verändert werden, andererseits höchst lichtempfindliche, die schon nach einigen Minuten oder Sekunden verblassen oder ganz ausgebleicht werden. Und da nun die Lichtechtheit eines Farb- 
stoffes diejenige Eigenschaft ist, die ihm seine Brauchbarkeit für Färbezwecke sichert, und die auch seinen Wert bestimmt, so prüft der Farbenchemiker und Techniker alle Farbstoffe in dieser Richtung. Man darf annehmen, daß von den ca. 6000 künstlichen Farbstoffen wohl ein jeder auf sein Verhalten gegen Licht untersucht worden ist, und zwar nicht allein für sich, sondern auch unter denjenigen Bedingungen, unter denen er später Verwendung finden soll. Diese Prüfungen haben sehr bemerkenswerte Resultate ergeben. Man weiß, daß die Lichtechtheit eines Farbstoffes abhängt von dem Material der Faser (Indigo ist auf Wolle echter als auf Baumwolle, die Methylviolette bleichen am raschesten auf Stärkepapier, langsamer auf Gelatine und Eiweißpapier). Man weiß ferner, daß die Lichtechtheit abhängig ist von der Art der Farbstoffixierung, d. h. von dem Vorhandensein und der Beschaffenheit der Beize, ferner abhängig von den klimatischen Verhältnissen, dem Zustande der Atmosphäre, dem Feuchtigkeitsgehalt der Luft usw. Alles wichtige Ergebnisse, deren detaillierte Beschreibung und Registrierung viele tausend Seiten der Fachliteratur ausfüllt.

Aber das Interesse an solchen photochemischen Prozessen darf nicht erschöpft sein durch die Feststellung der Tatsache, da $B$ ein Farbstoff verändert wird, o b er schnell oder langsam , bleich t"

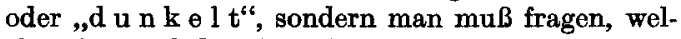
cher Art sind die Veränderungen, was entsteht aus dem Farbstoff, in welchem $\mathrm{Z}$ u s $\mathrm{t}$ a $\mathrm{nd}$ e befindet er sich $n$ a ch der Beliohtung? Was ist mit ihm geschehen? Ist er oxydiert, reduziert oder anderweitig verändert? Hierüber hat man erst in jüngster Zeit begonnen, exakte Versuche auszuführen, und hat sie auch auf viele andereStoffe ausgedehnt, die nicht Farbstoffe sind.

Solche Untersuchungen sind manchmal nicht ganz leicht auszuführen, da $\mathrm{d}$ a s $\mathrm{L}$ i ch $\mathrm{t} \mathrm{n}$ i c h $\mathrm{t}$ e in s e itig wirkt und da, wenigstens bei organischen Verbindungen, meist nicht ein einziges Lichtprodukt gebildet wird, sondern mehrere, die nicht immer leicht $\mathrm{zu}$ isolieren sind.

Man kann die Wirkungsweise des Lichtes niemals mit Sicherheit voraussagen; es führt uns von einer Uberraschung zur anderen. Spielend und in eleganter, unauffälliger Weise besorgt es zuweilen in kurzer Frist chemische Umsetzungen, zu deren $\mathrm{Zu}$ standekommen der Chomiker sonst eine lange Zeit braucht, eine große Folge von Reaktionen ausführen muBte, oder die er ohne das Licht überhaupt noch nicht zu realisieren imstande ist.

Alle diese Lichtreaktionen Oxydationen, Reduktionen, Polymerisierungen, Isomerisierungen, synthetische Prozesse, Spaltungen - lassensichin $z$ weigroBeGruppen teilen :

Erstens in solche, bei denen aus einer einzigen chemischen Verbindung oder durch Wechselwirkung mehrerer verschiedener Verbindungen andere Stoffe gebildet werden, die nunmehr sowohl im Lichte, als auch bei darauffolgendem Verweilen in der Dunkelheit stabil geworden sind. Hierher gehört z. B. die Oxydation des Jodwasserstoffes zu Wasser und Jod, die Reduktion des Chlors durch Wasserstoff $\mathrm{zu}$ Chlorwasserstoff, die Umlagerung von Maleinsäureester zu Fumarsäureester, die Polymerisierung von Jodacetylen, $\mathrm{C}_{2} \mathrm{HJ}$, zu Trijodbenzol, $\mathrm{C}_{6} \mathrm{H}_{3}$ usw. Solche Photoreaktionen, bei denen sich lichtla bile Stoffe oder Systeme dauernd in lich tstabile Gebilde verwandeln, sind, die weitaus überwiegenden. Und da solche einseitig verlaufenden , $\mathrm{n}$ i c h t umkehrbaren Reaktionen" sich tatsächlich auch freiwillig von selbst abspielen, so folgt daraus, daß das Licht in allen diesen Fällen nur Einfluß hat auf die $\mathrm{Ges} \mathrm{ch}$ w in dig k i t der Reaktion, daß es also P r o z e s se be s c h l e u$\mathrm{nigt}$, die auch ohne das Licht in allerdings viel längerer, manchmal nicht abzuwartender Zeit ihr Ende erreichen würden.

Die zweite Gruppe von Lichtreaktionen sind solche, bei denen einige Stoffe durch Belichtung in andere verwandelt werden, die dann ihrerseits bei darauffolgendem Verweilen im Dunkeln wieder umgekehrt in den ursprünglichen Zustand zurückkehren. Solche Vorgänge sind die ,u m k e h r baren Lichtreaktionen". Hierher gehören nur wenige Reaktionen, unter ihnen der von R. L u t h e r studierte Proze $\beta$ der Zersetzung des Halogensilbers und dessen Rückbildung aus Subhaloid und Halogen $2 \mathrm{AgCl} \rightarrow \mathrm{Ag}_{2} \mathrm{Cl}+\mathrm{Cl}$; die von demselben Forscher und F. W e i g e r t untersuchte Polymerisierung and Entpolymerisierung ron Anthracen und Dianthracen $2 \mathrm{C}_{14} \mathrm{H}_{10} \longrightarrow \mathrm{C}_{28} \mathrm{H}_{20}$; der Zerfall und die Rückbildung von Sohwefeltrioxyd $\mathrm{SO}_{3} \rightleftarrows \mathrm{SO}_{2}+\mathrm{O}(\mathrm{CoOh} \mathbf{n})$, die gegenseitige Umlagerung der beiden grauen krystallinischen Selenmodifikationen SeI $\rightarrow$ SeII (R. M a r c) und ferner eine Gruppe von Vorgängen, die mit dem Namen der Phototropieers chein ungen belegtwor. den sind, und die ich in jüngster Zeit an den F u 1 g i d e n nach zum Teil neuen Methoden studiert habe.

Die Fulgide werden nach einem von mir aufgefundenen Verfahren dargestellt. Sie haben die allgemeine Formel

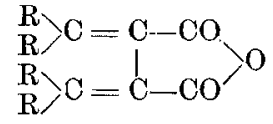

( $R$ bedeutet Wasserstoff, Alkyl oder Aryl) und kry stallisieren in prächtigen Gebilden von hohem Glanz und leuchtender gelber, orange, roter, purpurner bis brauner Farbe. Da sie durch das Licht entweder dauernd oder nur vor über g e h e $\mathrm{n} d$ verändert werden, eignen sie sich in ausgezeichneter Weise zu einer etwas eingehenderen Erläuterung der beiden eben charakterisierten, ,nicht umkehrbaren" und der ,umkehrbaren“" Photoreaktionen.

Bei ihren ,nicht umkehrbaren Photoreaktionen" entstehen aus den Fulgiden farblose oder hellfarbige, anders konstituierte Stoffe, die unter keinen Umständen weder im Lichte, noch im Dunkeln wieder in das ursprüngliche Fulgid zurückverwandelt werden können. Ich habe diese Lichtprodukte "Ph o to a n h y dride" genannt und. werde in kurzer Zeit ausführlich über sie berichten.

Bei ihren ,umkehrbaren Photoreaktionen" entstehen aus den Fulgiden tieferfarbige, gleich konstituierte Stoffe, die nur im Lichte stabil sind, und 
die sich aber in der Dunkelheit wieder rückwärts zu dem ursprünglichen Fulgide umformen.

Welche der beiden Reaktionen sich an einem Fulgide vollziehen, oder - präziser ausgedrückt welche von beiden Reaktionen überwiegt, hängt ab von der Dauer der Lichteinwirkung und von vielen anderen Faktoren. Ich schildere diese etwas komplizierten Verhältnisse am besten an einem besonders typischen Beispiele ${ }^{1}$ ), dem Triphenylfulgid

$$
\begin{aligned}
& \left(\mathrm{C}_{6} \mathrm{H}_{5}\right)_{2} \cdot \mathrm{C}=\mathrm{C}-\mathrm{CO} \\
& \mathrm{C}_{6} \mathrm{H}_{5} \cdot \mathrm{CH}=\mathrm{C}-\mathrm{CO}^{\prime} \mathrm{O} .
\end{aligned}
$$

Wird das für die Dunkelheit stabile orangegelbe Pulver dieses Stoffes zwischen zwei Glasplatten gepreBt, zur Hälfte schwarz verhüllt und zur anderen Hälfte einige Minuten von der Sonne oder von elektrischem Bogenlichte bestrahlt, so nimmt die belichtete Fläche eine hellbraune Farbe an, die aber bei darauffolgendem eintägigen Verwejlen in der Dunkelheit wieder in das ursprüngliche Orange ïbergeht. Diese phototrope Zustandsünderung kann man bei abwechselnder Belichtung und Verdunkelung sehr häufig, jedoch nicht unbegrenzt oft beobachten. Man findet nämlich bei mehrfacher Wieder-

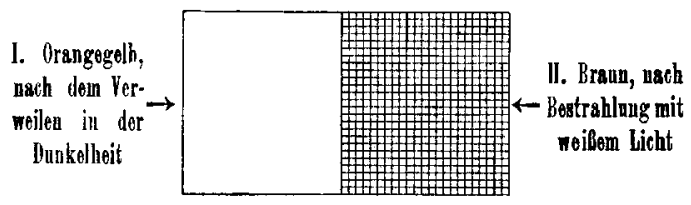

Fig. 1.

holung dieser Operation, daß die Nuancendifferenz zwischen der belichteten und nichtbelichteten Fläche immer kleiner wird, daß ferner das Hellbraun bei mehrtägiger konstanter Insolation immer mehr verblaßt, daß die belichtete Fläche allmählich heller wird als die unbelichtete, und daß schließlich das ganz hellgelb gewordene Pulver seine Farbe bei verschiedener Intensität der Beleuchtung nicht mehr wechselt. Es sind in diesem Falle aus dem ursprünglichen lichtlabilen Fulgide die farblosen licht- und dunkelstabilen Photoanhydride entstanden.

Wir erkennen also, wie bei der Belichtung der Fulgide zwei Reaktionen nebeneinander herlaufen, und wie die irreversible Reaktion schlieBlich die reversible, phototrope Reaktion verdeckt und ablöst.

Eine eingehende optische Analyse speriell der Phototropieers c h e in u n g e n hat nun zu Ergebnissen geführt, die zum Schluß in aller Kürze geschildert werden sollen $^{2}$ ). Wir sahen, wie das zur Hälfte schwarz verhüllte orangegelbe Fulgidpulver (I) auf der mit weißem Licht bestrahlten Fläche (II) hellbraun gew orden war (vgl. Fig. 1).

Läßt man nun für den weiteren Versuch die Fläche I verhüllt, bestrahlt die eine Hälfte von II

1) Die weiteren Ausführungen wurden von dem Vortragenden durch Experimente erläutert.

2) Die ausführliche Beschreibung dieses Gegens tandes siehe bei H. Sobbe, Ann. d. Chem. 359. weiter mit weifem Licht, die andere Hälfte von II (also ein Viertel des Ganzen) unter Kobaltglas mit blauem Licht, so verdunkelt sich auf der letzteren Fläche (III) die Farbe bis zum tiefsten Schwarzbraun (vgl. Fig. II).

Hieraus ergibt sich, daß unter blauem Lichte eine stärkere Einwirkung stattfindet, als unter weifem Lichte. Diese auf den ersten Blick merk würdige Tataache war nur so zu erklären, $\mathrm{da} B$ das weiße kombinierte Licht Strahlen enthalten muß, welche den blauen Strahlen entgegenarbeiten. Und in der Tat lehrt ein einfacher Versuch, daB das durch weißes und durch blaues Licht, ,e r r e g t e $\mathrm{e}^{\text {“6 }}$ Fuigid auf Fläche II und III bei Bestrahlung mit rotem und gelbem Lichte ebenso wie durch die Dunkelheit zu dem ursprünglichen orangegelben Fulgide, ,a u f g e h e 11 t" wird, daß also unter diesen Bedingungen kein Farbenunterschied zwischen den drei Flächen I, II und III zu erkennen ist.

Nimmt man nun an, daß den verschiedenen Farbennuancen eines erregten Fulgides nicht jedesmal eine verschiedene Modifikation entspricht, sondern daß das Triphenylfulgid überhaupt nur in $\mathbf{z} \mathbf{w}$ e i verschiedenfarbigen Formen existiert - in

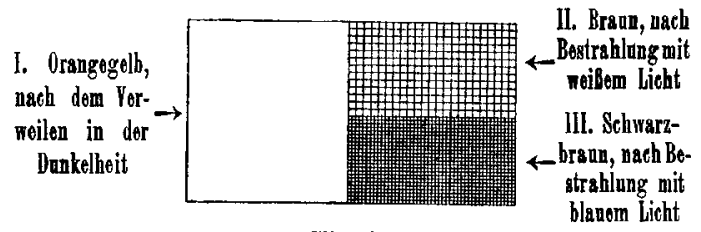

Fig. 2.

einer für die Dunkelheit farbenkonstanten hellsten Form $\mathrm{A}$ und in einer nur im Lichte stabilen dunkelsten Form B - so gilt folgende Beziehung:

unter blauem Lichte

Orange Fulgid A $\longrightarrow$ Schwarzbraunes Fulgid B. unter rotem $u$. gelbem Lichte

Das Fulgid A ist blauempfindlich, also gerade reaktionsfähig unter denjenigen strahlen, dieegselbst absorbiert (Bestätigung des Grotthusschen Gesetzes). Das Fulgid B is t rot-und gelbempfindlich, wird daher vornehmlich auch nur diese Strahlen absorbieren und in Wahrheit einen blauen Farbenton haben. Seine schwarzbraune Farbe ist nur eine Scheinfarbe, die dadurch bedingt ist, daB der blaue Stoff B noch mit unverändertem orange Stoff A gemischt ist.

Zwischen den beiden Fulgidmodifikationen besteht für jede Wellenlänge des Lichtes ein ,photoohemisches Gleichgewicht", eines jener stationären oder dynamischen Gleichgewichte, die zuerst von R. L $u t h$ e $r$ studiert worden sind. Sie sind verschieden von den wahren statischen Gleichgewich. ten, insofern sie nur so lange unverändert bleiben, als dauernd Licht von bestimmter Wellenlänge strahlt. Außer von der Wellenlänge des Lichtes ist das Gleichgewicht der beiden Fulgidformen ferner abhängig von der Lichtintensität und der Temperatur. - 
Ebenso wie das Triphenylfulgid sind in mehr oder weniger auffälliger Weise noch 19 andere Fulgide phototrop; ferner auch einige wenige andere Stoffe, z. B. die beiden von W. M a r c k w al d als phototrop erkannten Verbindungen, das salzsaure Chinochinolin und das B-Tetrachlorketonaphthalin

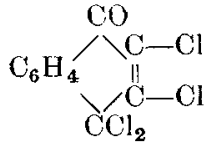

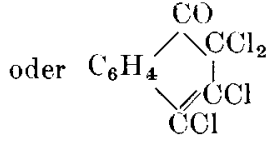

und schließlich auch die von $H$. B iltz untersuchten Phenylhydrazone einiger aromatischer Aldehyde und mehrere Osazone der Benzilreihe. Alle diese Stoffe sind von mir und meinem Mitarbeiter $\mathrm{R}$ i chard $\mathrm{H}$ ärtel nach den für die Fulgide ausgearbeiteten Methoden untersucht worden. Wir haben festgestellt, daß auch ihre Phototropieerscheinungen ganz denselben Gesetzen unterworfen sind, die ich für die Fulgide gefunden habe. So wird z. B. das weiße Tetrachlorketonaphthalin nur durch die ultravioletten Strahlen zu einer rotvioletten Modifikation e r r e g t, weil es als weißer Stoff nur diese Strahlengattung absorbiert. Und umgekehrt wird die rotviolette Modifikation dieses Ketons besonders schnell durch die gelben und grünen Strahlen zur weißen Modifikation a $\mathbf{u} \mathbf{f} \mathrm{g} \mathrm{e}$ $\mathrm{h}$ e $1 \mathrm{lt}$, weil es gerade diese absorbiert. Wir haben also :

unter ultraviolettem Licht

Weißes Keton A $\rightleftarrows$ Rotviolettes Keton B unter gelbem u. grünem Lichte

Das für die Dunkelheit farbenkon santeweiBeKetonA istultraviolett. empfindlich, das rotvioletteKeton B ist gelb und grün empfindlich.

Diese Beispiele mögen genügen. Wir sahen, daß sowohl beim Triphenylfulgid als auch beim Tetrachlorketonaphthalin eine besondere Gruppe von Strahlen die B-Formen in kürzester Zeit zu den A-Formen aufhellen, daß sie also einen Vorgang beschleunigen, der auch sonst bei Abwesenheit aller Lichtstrahlen in der absoluten Dunkelheit freiwillig von selbst verläuft.

Das letztere ist besonders wichtig. Das Licht leistet bei der Erregung eines phototropen Stoffes chemische Arbeit, indem es aus einem für die Dunkelheit stabilen Stoffe A einen lichtstabilen Stoff B schafft. Dieser Stoff B ist aber nur so lange haltbar, als Licht leuchtet; er gibt die in ihm aufgespeicherte Energie im Dunkeln ganz von selbst wieder ab unter Rückverwandlung in A. In welcher Form die Energie frei wird, kann ich noch nicht mit Bestimmtheit sagen. So viel steht aber fest, daß jeder phototrope Stoff ein Akkumulator für Lichtenergie ist, und daß die Zustandsänderungen eines solchen Stoffes, gerade so wie andere umkehrbare photochemische Prozesse geeignet sind, die Gesetze zu erforschen, die die Umwandlung der strahlenden Energie in andere Energieformen beherrschen.

\section{Über die Anwendung des Ultramikroskops nach Siedentopf \\ und des Mikrospektralphotometers nach Engelmann \\ in der Textil- und Farbstoff=Industrie. \\ Von N. Gaidukov.
(Eingeg. d. 20.11. 1908.)}

I. Die Anwendung des U1 tra $\mathrm{mikroskops}$.

Die mechanischen Methoden zur Prüfung der Spinnfasern (z. B. die Reißmaschine) zeigen nur die Eigenschaften der Fasern, aber nicht die Ursachen dieser Figenschaften. Auch das gewöhnliche Mikroskop kann diese Eigenschaften nicht zeigen, weil man mit Hilfe dieses Apparates meistens nur die äuRere Gestalt der Fasern, aber nicht die innere Struktur derselben beobachten kann. Deshalb ist es begreiflich, daß nach dem Erfinden des neuen mikroskopischen Apparates, in dem das Sichtbarmachungsvermögen stark gesteigert ist - nämlich des Ultramikroskopes -, auch Versuche gemacht wurden, diesen Apparat auch für die Untersuchung der Fasern anzuwenden.

Im Juli 1906 habe ich die präparierte Ramiefaser mit Hilfe des Ultramikroskopes nach $\mathrm{S}$ i e dentop $\mathrm{f}^{\mathbf{1}}$ ) beobachtet und habe gefunden, daß die Substanz dieser Fasern aus in Längsrichtung gelagerten Teilchen besteht ${ }^{2}$ ).

Herr Dozent J. S ch n e i d e r und Herr G. $\mathrm{K}$ u n z l - Prag ${ }^{3}$ ) haben für die Untersuchung gefärbter und ungefärbter Spinnfasern eine andere ultramikroskopische Einrichtung angewandt, das Ultramikroskop nach $H$. S i eden to p f und R. $\mathrm{Z}$ s i g m o n d y $\left.{ }^{4}\right)$. Dieser Apparat ist sehr geeignet für die Untersuchung flüssiger oder fester durch sichtiger kolloidaler Körper (Gold- und Silberhydrosolen, Eiweißlösungen, gefärbter Gläser und Steinsalze usw.), aber nicht für die Untersuchung der Objekte zwischen Objektträger und Deckglas. Dar$\mathrm{um}$ ist die Methode von $\mathrm{S} \mathrm{chnejder-Kunzl}$ zu kompliziert.

Ende Januar 1907 fragte mich Herr Ing. H e 1 l m a n n - Wien, ob man die Baumwollfasern mit Hilfe des Ultramikroskopes nach $\mathrm{S}$ i ed e $\mathrm{n}$ t o p f prüfen könne. Durch diese Frage angeregt und von der Firma $\mathrm{C}$ a $\mathrm{r} \perp \mathrm{Z}$ e i $\beta$ unterstützt, habe ich im Frühjahr 1907 im Mikrolaboratorium der Firma Carl Zeiß begonnen, das Problem zu lösen, ob man mit Hilfe des Ultramikroskops nach Siedentopf die Spinnfasern untersuchen und prüfen kann.

Als Arbeitshypothese für meine Untersuchungen habe ich die Micellartheorie von K. v. Nägeli ${ }^{5}$ )

1) C a r 1 Z e i B, Jena, Prospekt M i k r o 228

2) Berichte d. Deutsch. Botanisch. Gesellsch. 24, 587 (1906)

3) Z. f. wissenschaftliche Mikroskopie 23, 39; bis 409 (1906).

4) Car l Zei B, Jena, Prospekt M i k r o 229.

5) Nägeli und Schwendener, Das Mikroskop, 1877, 422-426. 